\title{
Carnets
}

Revue électronique d'études françaises de l'APEF

Première Série - 4 | 2012

(Res)sources de l'extravagance

\section{Forcer le trait. Caricature et la construction du personnage chez Michel Houellebecq: aperçu de la réception critique}

José Domingues de Almeida

\section{OpenEdition}

Journals

Édition électronique

URL : http://journals.openedition.org/carnets/7020

DOI : $10.4000 /$ carnets.7020

ISSN : 1646-7698

Éditeur

APEF

Édition imprimée

Date de publication : 1 janvier 2012

Pagination : 211-223

Référence électronique

José Domingues de Almeida, «Forcer le trait. Caricature et la construction du personnage chez

Michel Houellebecq: aperçu de la réception critique », Carnets [En ligne], Première Série - 4 | 2012, mis en ligne le 20 juin 2018, consulté le 01 mai 2019. URL : http://journals.openedition.org/carnets/7020 ; DOI : $10.4000 /$ carnets. 7020

\section{(c) (†) \&)}

Carnets est mis à disposition selon les termes de la licence Creative Commons - Atribution - Pas d'utilisation commerciale 4.0 International. 


\title{
FORCER LE TRAIT
}

\section{Caricature et la construction du personnage chez Michel Houellebecq: aperçu de la réception critique ${ }^{1}$}

JosÉ DOMINGUES DE ALMEIDA

Université de Porto - ILC - Margarida Losa

jalmeida@letras.up.pt

\begin{abstract}
Résumé
Une des caractéristiques, parmi d'autres tout aussi polémiques de l'écriture romanesque houellebecquienne, tient à l'extravagance et l'exagération que ce romancier très médiatique met dans la simple évocation des personnages et dans la fonction qu'il assigne à ces caricatures. Ce faisant, ce que nous entendons mettre en lumière dans cet article -, il s'agit avant tout chez lui, par le truchement de la fiction, de fournir une réflexion sur des constructions sur des catégories sociétales et de porter un regard subjectif impitoyable sur certains acteurs sociaux, comme autant d'ennemis à abattre ou à critiquer par la fiction.
\end{abstract}

\section{Abstract}

One the most important features of Michel Houellebecq's novelistic writing is extravagancy and exaggeration. We will try to show how this writer builds his characters through cliché and epiphrastic reflection. We would like to highlight in this article the way this author, by fictional means, considers some social categories and actors by emphasizing with ruthless prejudice some of their features as though they were somehow enemies to be defeated.

Mots-clés: Michel Houellebecq, littérature, provocation, extravagance, épiphrase Keywords: Michel Houellebecq, literature, provocation, extravagancy, epiphrasis

\footnotetext{
${ }^{1}$ Cet article a été élaboré dans le cadre du projet "Interidentidades" de L'Institut de Literatura Comparada Margarida Losa de la Faculté des Lettres de l'Université de Porto, une I\&D subventionnée par la Fundação para a Ciência e a Tecnologia, intégrée dans le "Programa Operacional Ciência, Tecnologia e Inovação (POCTI), Quadro de Apoio III (POCTI-SFA-18-500).
} 
La contemporanéité littéraire française s'avère trop fertile en coups médiatiques pour que la critique ne se montre pas vigilante et fasse la part des choses entre ce qui tient de l'écriture littéraire proprement dite et de l'inauthentique plagiat d'états d'âmes ou de sautes d'humeur, tant le risque d'enlisement bon marché pointe à chaque tour de page dans l'écriture du jour (Cf. Viart \& Vercier, 2005: 8s). C'est à cet équilibre intelligent que s'était essayé Christian Prigent avec une rare et exigeante acuité de lecteur désabusé et averti à qui on les fait pas / plus. II s'agit d'entrée de jeu pour ce critique de séparer une littérature que Viart \& Vercier qualifient de consentante, ou encore concertante, d'avec celle qui compte et qui "merdre" (1991), et qu'il préfère.

Dans la lignée ou la foulée d'un Rabelais, Lautréamont, Sade, Roche, Guyotat, Verheggen ou encore Novarina, - vaste tradition du littérairement incorrect et d'une approche insolente de l'écriture fictionnelle -, il lui est pénible de lister de nouveaux noms, tant le panorama actuel, marqué par ce dramatique "oubli du moderne" (ib.,: 9-29) s'inscrit plutôt dans l'inoffensif et le feutré: "Le drame serait que les chromos chics viennent laver ce qu'Artaud appelait la 'cochonnerie' d'écriture. Le drame serait que le silence se fasse sur cette de bêtise obstinée, cette croyance, cette folie qui fait socle éthique pour l'aventure dans la langue $[\ldots] "$ (ib.,: 17).

Et pourtant, quelques noms et ouvrages s'inscrivent en faux dans ce paysage désolant, parmi lesquels il faut compter Millet, Savitzkaya (première plume), Murray et quelques autres... Et Michel Houellebecq, écrivain que l'on ne présente plus, et qui s'est vu décerner le Prix Goncourt pour La carte et le territoire (2010), mais très certainement, de façon implicite, pour l'intégralité de son œuvre et de son projet littéraire. À cet égard, une biobibliographie du phénomène de l'écriture houellebecquienne a vite vu le jour en plusieurs essais qui ne se signalent pas tous par l'encensement, loin s'en faut! Rappelons les principaux: Murielle-Lucie Clément (2003), Jean-François Patricola (2005) ou Denis Demonpion (2005).

Mais là un bémol s'impose qui engage toute la complexité de la plume de Michel Houellebecq. Si son écriture peut bien s'inscrire dans la littérature concertante en ce qu'"elle fait chorus sur le cliché du moment et se porte à grand bruit sur le devant de la scène culturelle. Elle trouve dans ce bruit le seul gage de sa valeur car sa recherche est celle du 'scandale', mais il s'agit d'un scandale calibré selon le goût du jour, 'surfant' sur le goût que le jour peut avoir, par exemple, pour les jeux du sexe, du spectacle ou du cynisme" (Viart \& Vercier, 2005: 9), elle vise donc plus loin.

La critique et les instances de légitimation littéraires reconnaissent chez lui un travail spécifique de la langue et de la fiction qui vaut le détour et qui ne laisse en tous cas personne indifférent. Est-ce là le but, ou en tous cas le critère d'une bonne écriture au sens 
prigentien, un gage de qualité littéraire qui prévient le bâillement généralisé contemporain? Nous sommes tenté de le penser à lire toutes les réactions qu'incite chaque parution.

Michel Tournier, qui décrit l'écriture romanesque houellebecquienne comme un "un revolver sur la tempe" (Gandillot, 2006), a eu beau essayer de lui "fai[re] rater le Goncourt" (ib.,), cette fois-ci, ça n'a pas marché. En cause, chez certains critiques, la fameuse "platitude" du style de Michel Houellebecq que Jean-François Patricola décline en spécialiste de l'auteur primé: "[...] sa langue est apathique, flegmatique et dépitée, banale, proche du slogan publicitaire ou de la harangue, voire de l'insulte, commune et partagée par tous" (2005: 220); une critique qui rejoint l'appréciation d'autres lectures plus généralistes ou dépressives: "La platitude du style ou sa crudité prétendent être en prise directe sur une époque qui renonce à toute élégance. Au mieux platitude et crudité s'affirment 'cliniques', sans voir qu'elles contribuent à leur tour à dégrader le tableau qu'elles dressent" (Viart \& Vercier, 2005: 352); "Point de vue plat et morne, c'est réussi [...]" (Huston, 2004: 291).

Si Houellebecq ne cache pas son penchant pour l'"aphorisme facile", non sans un humour grinçant ou faussement naïf qui laisse sur ses lecteurs ce petit sourire en coin, mais au risque de la "platitude", comme par exemple: "Adolescent, Michel croyait que la souffrance donnait à l'homme une dignité supplémentaire. II devait maintenant en convenir: il s'était trompé. Ce qui donnait à l'homme une dignité supplémentaire, c'était la télévision" (Houellebecq, 1998: 120), ou encore ces conclusions en queue de poisson: "En ce sens, les serials killers des années quatre-vingt-dix étaient les enfants naturels des hippies des années soixante; on pouvait trouver les ancêtres communs chez les actionnistes viennois des années cinquante" (ib.,: 211). D'autres tirades frisent même le mauvais goût provocateur: "Tu sais comment on appelle le gras qu'il y autour du vagin? Non. La femme" (Houellebecq, 2005: 22).

II n'est dès lors pas étonnant qu'un pan de la critique n'aient pas hésité à relier directement cette plume à une mouvance plus vaste qui se trouve à l'œuvre depuis la fin déclarée de la textualité, - Ch. Prigent ne dédie-t-il pas son essai nostalgique à "mes amis de TXT"? -, et qui assume la taxinomie de la "tradition de l'insolence" (Cf. Blanckeman, 2000) et compte des noms comme Marie Darrieusecq, Amélie Nothomb ou Christine Angot, dont les romans s'assurent d'ailleurs de bonnes parts du marché littéraire en francophonie ou à l'étranger, le marché de la traduction aidant.

C'est cet aspect extravagant, c'est-à-dire expressément "réactionnaire" chez Michel Houllebecq, qui mérite d'être creusé dans les textes proprement dits, mais également au vu de ce qui s'est écrit dans l'escorte critique qui les a suivis. II s'agit en tous cas de forcer le trait à la faveur de la caricature dégagée par la caractérisation acharnée de certains personnages. Ce qui ressort des points de contact, le plus souvent discordants, entre 
l'écriture houellebecquienne et une certaine tendance de l'essai contemporain, vigilant ou inquiet, c'est un malaise personnel et sociétal qui n'ose pas dire son nom, mais qui trouve son origine dans l'héritage de Mai 68 en tant que révolution axiologique.

La fiction de Michel Houellebecq et l'auteur lui-même, du fait des données biographiques, si l'on en croit ses biographes adulateurs ou détracteurs, se trouvent donc placés dans, ou reflètent à leur façon, le débat post-Mai 68 tel qu'il s'est tenu lors des dernières élections présidentielles françaises, qui donnèrent la victoire à Nicolas Sarkozy, et tel qu'il a fini par se traduire en arguments échangés dans la presse et dans l'essai ( $C f$. Weber, 2008). On remarquera, à cet égard, les tangences, même ironiques et dépréciatives, chez (les personnages mis en scène par) Houellebecq et le discours de l'essai des défenseurs de l'héritage soixante-huitard.

Si Daniel Lindenberg (2002) a expressément intitulé sa contribution "Le rappel à l'ordre", c'est pour s'insurger contre une offensive généralisée et "réactionnaire" contre tous les acquis d'une culture et d'une civilisation née sur les pavés de Paris aux alentours de Mai 68 qui menace les assises du destin collectif français, son épopée républicaine issue de la Révolution. Houellebecq y revient indirectement: "Le mouvement de mai 1968 l'avait impressionné, et au moment où la vague hippie commença à refluer en Californie il se dit qu'il y avait peut-être quelque chose à faire avec la jeunesse européenne" (Houellebecq, 1998: 81).

Et Lindenberg de préciser la visée de son pamphlet. II s'agit de dénoncer tous les "procès" instaurés à la faveur d'idéologies révisant dangereusement l'Histoire ou contestant des avancées sociales que l'on tient pour acquises, mais que des discours réactionnaires seraient en train de tempérer, voire "réviser" sous couvert de "néoconservatisme", "populisme" ou "national-républicanisme"; en tous cas avec un cynisme affiché (2002: 9). Ces discours tiennent ces acquis pour responsables des frustrations personnelles et des désagrégations sociales.

Or, un de ces procès vise précisément l'auteur de Les particules élémentaires, accusé d'endosser, via ses personnages, le mépris à l'égard du "[...] tourisme de masse comme métaphore de la décadence contemporaine" (ib.,: 20); une conception illustrée par le roman Plateforme, récit alliant la critique explicite de la massification des loisirs au tourisme sexuel, et donc à une certaine "misère sexuelle, absence d'amour, aspiration au suicide, horreur du passage du temps et de la mortalité" (Huston, 2004: 294). Cette misère taraude l'homo occidentalis, espèce en décadence ou en voie d'extinction, impuissant ou indécis sexuel laissé pour compte et menacé par une idéologie décaractérisante et assimilatrice à outrance dans laquelle il perd ses repères. C'est d'ailleurs tout le malheur identitaire de l'antihéros de Plateforme aux prises avec son insécurité et voué dès lors à l'expérimentalisme sexuel pur et dur (Houellebecq, 1998: 122ss). 
Ces symptômes ne sont pas passés inaperçus à certains essayistes, conscients de ce que Houellebecq force le trait à la faveur de ses personnages, alter egos de ses déboires personnels. Pour Daniel Lindenberg, un tel discours atteste du "mépris du touriste, qui vise ici 'les classes moyennes' [et qui] prend pour cible une forme de loisir qui s'est continuellement démocratisée depuis le Front Populaire" (Lindenberg, 2002: 21). Par ailleurs, cet auteur s'en prend au procès houellebecquien de la liberté des mœurs en tant qu'acquis majeur du Mai 68 français. Associez cette critique aux menaces qui guettent ou minent déjà le mâle houellebecquien et vous comprendrez mieux les torts qu'on lui impute:

Une grande partie de la société (plutôt les mâles, âgés et ouvriers-employés, ou encore catholiques pratiquants) n'acceptent toujours pas ce que les sociologues appellent le 'libéralisme culturel' En particulier lorsque les valeurs viriles et la 'domination masculine' sont mises en cause. C'est le talent d'un Houellebecq d'avoir senti ce malaise [...] (ib.,: 23).

La crise du mâle, en mal d'identité, en manque de puissance symbolique et de ritualisation initiatique en vue de son affirmation phallique individuelle et sociale est évidente. Ce mâle fragile, en tous cas en devenir, décrit par le pertinent essai d'Elisabeth Badinter (Cf. Badinter, 1992), aspire à s'assurer un statut dans la tribu postmoderne et post-soixantehuitarde. II réclame, souvent puérilement, son rôle défensif et territorial dans cette même tribu contre la menace de l'Autre non français, noir, arabe, étranger. Ce désir ou revendication de puissance passe aussi par une mimique de la domination des femmes; une allégorie de l'incapacité pour le mâle à se définir face à la femme émancipée et agressive: "La plupart des hommes préfèrent les pipes, dit-elle encore. La pénétration les ennuie, ils ont du mal à bander. Mais quand on les prend dans la bouche ils redeviennent comme de petits enfants. J'ai l'impression que le féminisme les a durement atteints, plus qu'ils n'ont voulu l'avouer" (Houellebecq, 1998: 140).

On comprend mieux, dès lors, que ce narrateur mâle force le trait quand il s'agit de se référer aux femmes soi-disant libérées et qu'il ne les ménage pas (ib.,: 121). Lindenberg y lit "le retournement de certaines théories féministes extrêmes" (Lindenberg, 2002: 24). Même désarroi à l'étranger à l'égard de coups portés au discours soixante-huitard et à une certaine image de l'Hexagone. C'est le cas de Perry Anderson:

L'idéologie des droits de l'homme, toute libérale qu'elle parût - n'avait-elle pas été le plat de résistance du banquet idéologique du Bicentenaire? -, ne constituait pas une politique. Succédané contemporain de ce qui avait été autrefois les idéaux du socialisme, elle sapait la cohérence de la notion en tant qu'être collectif, et elle 
aboutissait à des exigences intrinsèquement contradictoires: droit à l'égalité et droit à la différence proclamés dans un même souffle (Anderson, 2005: 59).

À nouveau, l'essai accuse "le romancier aujourd'hui à la mode Michel Houellebecq le "Baudelaire des supermarchés"' (ib.,: 28) et dénonce "le débit monotone de ses phrases sans relief ni vigueur [...] censé reproduire le monde démoralisé qu'elles dépeignent [...]" (ib.,). En fait, c'est toujours un rapprochement subtil auteur-narrateur-personnage qui est convoqué pour justifier un argumentaire aux relents néoconservateurs et une manie de la caricature du personnage afin de ridiculiser les effets concrets de l'idéologie droit-deI'hommiste. D'où aussi le titre très dépréciatif du chapitre "Michel Houellebecq" chez Nancy Huston, intitulé “L'extase du dégoût: Michel Houellebecq" (Huston, 2004: 279 - 300).

À la suite du Prix Goncourt 2010 remporté par cet écrivain, les pincettes avec lesquelles Huston prenait ce dossier en mains semblent bien périmées: "Michel Houellebecq est-il un écrivain important? Mérite-t-il de figurer aux côtés de Beckett et de Cioran, de Bernard et de Kundera? Je ne sais pas. Mais, vu l'attention médiatique qu'il a suscitée depuis une dizaine d'années et le fait qu'en 2002 il a reçu pour Atomized (Les particules élémentaires) le très prestigieux prix Impac [...] il me semble digne d'intérêt" (ib.,: 279). Et Huston de le qualifier de "l'un des champions les plus ardents de la philosophie du désespoir à l'époque contemporaine" (ib.,). Comme elle le fait pour d'autres écrivains tout aussi dépressifs selon elle, Huston exhume une biographie conjecturelle enfouie: "On peut supposer que l'écriture a donné à Houellebecq [...] la possibilité de dire et de combattre en même temps un mal de vivre intense" (ib.,: 281), même si Donald Morrison considère quand même Michel Houellebecq "une cible facile" (Morrison, 2008: 55) pour la critique!

Pour Huston, l'œuvre houellebecquienne relève davantage du voyeurisme que de la dénonciation de sévices passés et endurés. Elle implique la poursuite d'un projet littéraire qu'elle rappelle: "détruire" (Houston, 2004: 289). C'est-à-dire "éliminer les détails. Rejeter la complexité. Mettre en scène des personnages lambda, mous et passifs, fades, neutres et écœurés. La grande originalité de Houellebecq consiste à braquer ses projecteurs romanesques sur le monde du banal' (ib.,). On est assez proche du "roman à thèse", mais quelles thèses? Et puis comment les faire dire aux personnages ou au texte si ce n'est par le recours à la caricature et au trait forcé.

Pierre Jourde, dont le souci de l'authenticité en littérature a suscité un essai très profond (2005), considère l'écrivain Michel Houellebecq un "individu louche" dont il parle dans un autre livre décapant sur le roman français contemporain (2002: 217), mais qu'il évite expressément de confondre avec les autres noms d'auteurs associés aux extravagances médiatiques du moment et à ce que nous nommions plus haut "la tradition de l'insolence". Comme il le souligne lui-même: "Rien à voir avec le projet de Houellebecq, par ailleurs 
nettement réactionnaire" (ib.,: 218). Nuance, donc! Cette attitude trahit en fait la réception positive d'une partie de la critique littéraire, notamment naturaliste, qui voit dans le travail fictionnel houellebecquien une tentative originale de renouer avec le réel social et qui se traduira très symboliquement par l'attribution du Prix Goncourt en 2010.

Contrairement à Nancy Huston, Jourde reconnaît qu'"un personnage n'est pas son auteur, mais une figure possible de sa personnalité, une potentialité qu'il a plus ou moins développée dans la réalité" (ib.,: 224). Toutefois, force est de constater qu'il s'établit un rapport certain entre pensée de l'auteur et comportement ou discours du personnage dont la nature fait d'ailleurs l'objet d'une fine analyse de Jean-François Patricola. Ce critique met à nu la stratégie narrative houellebecquienne qu'il affirme reposer sur le recours à deux figures de style permettant de faire tenir aux personnages et aux textes, avec une distance ou un détachement faussement assumés, des propos ou des clichés tout en évitant l'association et la paternité, et en brouillant les pistes en cas de poursuites.

Patricola parle d'une "rhétorique de l'assimilation, de la capillarité et de l'insinuation, de la juxtaposition, qu'elle soit directe ou indirecte, par des figures stylistiques identifiables" (Patricola, 2005: 264). Ce critique rabat-joie en détecte deux qu'il considère récurrentes, et qui permettent à l'écrivain l'extravagance caricaturale dans l'élaboration du personnage. D'une part, l'épiphrase qui "agit comme une parenthèse, une didascalie dans le récit" (ib.,) et, d'autre part, la parataxe, comme procédé systématique de simplification des choses ou des théories, et dès lors, comme évitement de la complexité dont parle Huston. Les particules élémentaires (1998) ou encore La carte et le territoire (2010) recourent spontanément à ce mécanisme stylistique par lequel les personnages épousent les points de vue de l'auteur tout en assumant les responsabilités de l'écrivain. Cependant, son effet provocateur va perdant de son intensité jusqu'à en devenir inoffensif ou attendu, si l'on en croit l'accueil réservé à ce dernier roman.

Rappelons très brièvement le contenu diégétique de ces deux textes. Le premier roman narre les mésaventures de deux frères, Michel et Bruno, tous deux héritiers et victimes des prétendus déboires post-Mai 68: mère libérée, divorce, misère sexuelle, incapacité à établir des rapports sociaux, new age et déprime généralisée. Les "particules élémentaires" sur lesquelles Michel planche au CNRS procurent une forte métaphore de la coexistence sociale postmoderne, dont Gilles Lipovetsky n'a cessé de suivre le procès sous forme d'individualisme depuis L'ère du vide (1983).

Le second roman, qui n'hésite pas à mettre l'écrivain lui-même en scène par une sorte de mise en abyme égocentrée: "[...] tel était, souligne Houellebecq dans son texte, le profond paradoxe de cette toile" (Houellebecq, 2010: 192), évoque le parcours d'un artiste peintre, Jed Martin, fait d'une succession d'allers-retours dans le passé et l'avenir. Jed Martin sollicite les services de Houellebecq, installé en Irlande et lui demande de participer au 
catalogue de sa future exposition. II lui présente ses travaux et obtient son accord de principe et lui propose même de faire son portrait. Le vernissage s'avère un énorme succès qui lui vaut la gloire et la fortune dans le milieu hermétique du marché de l'art, même si le personnage principal n'arrive pas à communiquer et à interagir socialement. Le récit se termine par l'enquête policière sur le meurtre de Houellebecq, effroyablement assassiné chez lui. L'auteur imagine son propre enterrement au cimetière Montparnasse et Jed aide le commissaire Jasselin à résoudre l'affaire.

Les techniques narratives auxquelles nous faisions allusion plus haut investissent ces deux décors narratifs de déprime personnelle et sociale: "[...] il était nécessaire d'aller jusqu'au bout de l'autodépréciation, de contempler pleinement l'abjection de son ventre gonflé, de ses bajoues, de ses fesses déjà pendantes" (ib.,: 151). D’abord, dans Les particules élémentaires, il est question d'un monde sans Dieu: "Il s'étonnait de souffrir autant. Profondément éloignée des catégories chrétiennes de la rédemption et de la grâce, étrangère à la notion même de liberté et de pardon, sa vision du monde en acquérait quelque chose de mécanique et d'impitoyable" (ib.,: 89), comme cadre d'une société dont les "particules élémentaires" subsistent de façon entomologique (Cf. aussi Houellebecq, 2010: 258): "depuis des années, Michel menait une existence purement intellectuelle. Les sentiments qui constituent la vie des hommes n'étaient pas son sujet d'observation; il les connaissait mal. [...]. II observait [...]. Que conclure? Quelle interprétation donner à tous ces comportements? C'était difficile" (Houellebecq, 1998: 119).

Directement visée par les attaques de l'auteur, et ce par le biais des personnages, ce qui cautionne l'intuition d'un "roman à thèse" -: les mentalités libertaires nées dans la foulée des revendications de Mai 68 et des révolutions sexuelle et féministe qui ont eu pour effet de brouiller, voire d'inverser les rôles symboliques et sociaux: "Il est piquant de constater que cette libération sexuelle a parfois été présentée sous forme d'un rêve communautaire, alors qu'il s'agissait en réalité d'un nouveau palier dans la montée historique de l'individualisme" (ib.,: 116).

Résultat, côté lecteur: on accuse un vide, regrette timidement ou sarcastiquement une stabilité, mais finit par se complaire dans ce malaise déprimant. Nancy Huston a bien caractérisé l'état d'esprit du narrataire du roman à thèse houellebecquien: “C'est un tour de passe-passe extraordinaire: le lecteur, cautionné par les grandes envolées théoriques et pseudo-scientifiques de Michel Houellebecq, se sent supérieur, voire révolutionnaire, moyennent quoi il peut se laisser choquer et exciter par les personnages violents de provocation pure, comme un enfant de quatre ans par l'usage des gros mots [...]" (Huston, 2004: 300).

Aussi, les personnages entretiennent-ils des rapports très éphémères et rudimentaires et tiennent-ils des propos très "utilitaires" pour l'écrivain: "C'était peut-être une 
idée, se dit-il; entre voisins, on fait connaissance dans un camping; pas forcément pour baiser, mais on fait connaissance, c'est un démarrage possible" (Houellebecq, 1998: 99). Le moyen favori pour décrire et dénoncer cette société bloquée dans ses contradictions est le recours au cliché et au commentaire épiphrastique. La haine y rejoint le besoin d'humiliation de toute une galerie de catégories considérées d'un point de vue purement typologique.

La "femme libérée" fait l'objet de virulentes attaques misogynes. Elle se confond avec son cliché érotique interchangeable et sans cesse ressassé: "Elle avait de jolies fesses, encore bien rondes, très excitantes" (ib.,: 143), ou avec les fonctions anatomiques de son corps de "femelle", cliniquement évoquées: "A partir de l'âge de treize ans, sous l'influence de la progestérone et de l'œstradiol sécrétés par les ovaires, des coussinets graisseux se déposent chez la jeune fille à la hauteur des seins et des fesses. Ces organes acquièrent dans le meilleur des cas un aspect plein, harmonieux et rond [...]" (ib.,: 57).

La femme-femelle se montre dès lors immédiatement disponible pour satisfaire les besoins sexuels de l'homme, en mal d'affirmation et donc menacé. Elle constitue littéralement un objet sexuel dénué de toute intelligence ou jugement: "Il y avait une jeune Coréenne, très jolie, j'ai tout de suite eu envie de la sauter" (ib.,: 175), et son image active un fantasme irrésistible d'humiliation et de dégradation: "L'animatrice de l'atelier d'écriture avait de longs cheveux noirs, une grande bouche soulignée de carmin (de ce type qu'on appelle communément 'bouche à pipes'” (ib.,: 109). L'essentiel de la rhétorique houellebecquienne consiste en commentaires insidieux, latéraux, anodins, mais terriblement efficaces pour l'illustration passagère de la thèse qu'il s'agit de vendre à un lecteur friand de sensation de "supériorité" (Cf. Huston, 2004: 300).

Le garçon fait, lui aussi, l'objet d'une analyse entomologique. Ses comportements sont expliqués par une observation animale, grégaire: "La plupart des garçons, surtout lorsqu'ils sont réunis en bandes, aspirent à infliger aux êtres les plus faibles des humiliations et des tortures" (Houellebecq, 1998: 44), et un peu plus loin: "Les sociétés animales fonctionnent pratiquement toutes sur un système de dominance lié à la force relative de leurs membres. Ce système se caractérise par une hiérarchie stricte [...]" (ib.,: 45). Très proche du documentaire sur la vie animale, la didascalie textuelle, c'est-à-dire la voix sournoise de l'auteur, poursuit l'approche entomologique: "Cependant, l'animal le plus faible est en général en mesure d'éviter le combat par l'adoption d'une posture de soumission (accroupissement, présentation de l'anus)" (ib.,: 46).

Mais d'autres catégories servent également de cibles aux propos réactionnaires des personnages, ou faudrait-il plutôt dire de l'auteur? À nouveau, le cliché et l'épiphrase s'emparent des considérations émises. C'est le cas des Arabes et des Noirs, dont on fait l'amalgame par une logique de cause à effet. Le refus de toute mixité, si ce n'est l'accouplement fétichiste interracial, s'insinue à la faveur de ces parenthèses: "Ça surprend 
beaucoup de gens, mais Noyon est une ville violente. II y a beaucoup de Noirs et d'Arabes, le Front national a fait 40\% aux dernières élections" (ib.,: 148). Les Noirs, forcément, se signalent aux préjugés de l'auteur via le personnage par "une grosse bite" (ib.,: 195); c'est-àdire leur animalité.

À cet égard, le pamphlet raciste de Bruno permet à l'écrivain de le signer sans trahir sa présence. Les propos antisémites qui y figurent donnent à lire des idées latentes, qui font librement leur chemin: "Seuls les Juifs échappent au regret de ne pas être nègres, car ils ont choisi depuis longtemps la voie de l'intelligence, de la culpabilité et de la honte" (ib.,). Même recette pour les catholiques, "observés" de façon entomologique: "Pour le déjeuner, il repéra une catholique" (ib.,: 112), comme une catégorie prédéterminée et immédiatement identifiable, soumise à toutes sortes de poncifs. Et, à nouveau, le personnage permet à l'auteur d'émettre ses impressions personnelles: "Quoique catholique, la catholique n'aimait pas le pape; avec son mental moyenâgeux, Jean-Paul II freinait l'évolution spirituelle de l'Occident, telle était sa thèse" (ib.,), à savoir la thèse de l'écrivain.

Le narrateur réserve pareil sort, par le biais des personnages, aux homosexuels, décrits selon les attentes et les préjugés de l'écrivain. Ce troupeau facilement identifiable à de simples codes vestimentaire et rituels se voit assigné à un territoire grégaire défini, une espèce de "réserve" animale: "De l'autre côté de la Seine, sur le quai des Tuileries, des homosexuels circulaient au soleil, discutaient à deux ou par petits groupes, partageaient leurs serviettes. Presque tous étaient vêtus de strings" (ib.,: 18).

Mêmes procédés et même rhétorique dans La carte et le territoire même si, très nettement, et contrairement aux romans précédents, la critique et le lectorat houellebecquiens ont plutôt bien reçu ce texte envers lequel ils ne tarissent pas d'éloges ${ }^{2}$. La construction de clichés et d'une typologie des personnages, à vendre jouissivement au lecteur, est toujours active, mais l'effet cynique et dépréciatif ne joue plus à fond ou n'est plus ressenti comme tel par les lecteurs et surtout par la critique littéraire qui en vu / lu d'autres. En quelque sorte, en vue de, et dans ce roman, l'écrivain s'est réconcilié avec la critique et avec un lectorat qu'il aimait à scandaliser dans ses précédents textes, notamment dans Les particules élémentaires.

Olga, l'amante russe transitoire de Jed, nous est décrite selon un catalogage spontané: "Avec son teint pâle, presque translucide, ses cheveux d'un blond platine et ses pommettes saillantes, elle correspondait parfaitement à l'image de la beauté slave telle que l'ont popularisée les agences de mannequins et les magazines après la chute de l'URSS"

\footnotetext{
${ }^{2}$ II n'en faut pour preuve que le dossier que le magazine Télérama consacre à la sortie du livre, et ce avant même que ne lui soit décerné le Prix Goncourt. La critique littéraire, et plusieurs lecteurs, y saluent une plume nouvelle, moins scandaleuse, plus ironique et assagie d'autant plus que l'écrivain lui-même s'y est investi et mis en autofiction. (Cf. Télérama, août 2011). Mêmes réception et engouement après le Prix Goncourt. L'unanimité de la réception positive du roman est sans cesse mise en exergue (Cf.Télérama, novembre 2011).
} 
(Houellebecq, 2010: 64). Or, “[...] l'ère des mannequins anorexiques était bel et bien terminée, et les femmes exagérément plantureuses n'intéressaient plus que quelques Africains et quelques pervers [...]" (ib.,: 73). De même, "Comment se fait-il que les nouveaux cuisiniers [...] soient homosexuels?" (ib.,: 86). Un galeriste abordant Jed Martin prend vite l'aspect d'"un situationniste belge" ou d'"un intellectuel prolétarien" (ib.,: 110). Si "l'arrogance" (ib.,: 162) est caractéristique des Japonais, le déséquilibre hormonal l'est tout autant des adolescents espagnols, détectable à "leur taux d'hormones [...] incroyablement élevé" (ib.,: 364). Ou encore la réceptionniste de la clinique suisse, où le père du personnage principal s'est fait euthanasier, "ressemblait à une ménagère de l'Allgäu typique [...]" (ib.,: 373). Par ailleurs, l'une des pistes de l'enquête sur le meurtre de Houellebecq habite Nice et "On avait affaire au cadre de vie classique, prévisible, d'un grand bourgeois hédoniste et pas très raffiné [...]" (ib.,: 388).

Ces exemples sont légion dans le roman et qui attestent d'une intention de catégorisation selon des préjugés et des clichés sociaux bien définis qu'il s'agit de faire subtilement partager par le lecteur, qui, par ailleurs, se voit ébloui par le sens (de moins en moins) provocateur du commentaire. À nouveau, la logique de l'épiphrase et de la parenthèse discursive joue à fond chez le narrateur en guise de théorisation ou argumentaire sociologique. Franz, l'ami galeriste de Jed Martin, devenu riche, a du mal à gérer son nouveau statut, "comme c'est souvent le cas pour les gens issus d'un milieu pauvre [...]" (ib.,: 396).

Toute progression narrative devient l'occasion d'une parenthèse-didascalie où l'écrivain lâche son commentaire, son sabir social et politique, et essaie d'épater le lecteur par une compétence réflexive qui se superpose à l'élaboration narrative proprement dite. Alors, qui y a-t-il de si utile à ouvrir si souvent la parenthèse si ce n'est un souci réactionnaire d'avancer subtilement, bien à abri, derrière le bouclier du personnage, même si ces propos seront reproduits ailleurs en péritextes divers? Toutefois, il semblerait que le procédé se soit épuisé et que le bourgeois (critique littéraire et lecteurs en général) ne se laisse plus épater si l'on a croit l'impact plutôt consensuel du roman et de la reconnaissance et consécration par le Prix Goncourt.

En fait, dans La carte et le territoire, à nouveau, la plume clinique et réflexive à outrance s'impose toujours et fait très justement penser à la perpétuation d'un seul et même personnage. Nancy Huston n'y va pas par quatre chemins pour tourner en dérision cette manie: "N'est-il pas difficile de construire un roman avec des personnages qui sont tous pareils?" (Huston, 2004: 291). L'effet du texte se construit donc quelque part entre l'indigence psychologique des personnages renvoyés à leur cliché et la subtilité argumentative du narrateur dans la parenthèse. On y lit par exemple que "La peur du gendarme [...] était décidément la vraie base de la société humaine [...]" (ib.,: 294), que 
"Dans les pays latins, la politique peut suffire aux besoins de la conversation des mâles d'âge moyen ou élevé [...]" (ib.,: 22), ou encore que "Les seins siliconés sont ridicules lorsque le visage de la femme est atrocement ridé, lorsque le reste du corps est dégradé, adipeux et flasque [...]" (ib.,: 329). Houellebecq a en effet toujours le sens de l'aphorisme sans appel, même si ce dernier roman n'en fait plus un outil de provocation gratuite.

Cet aperçu du discours tenu par l'auteur et les personnages de Les particules élémentaires et de La carte et le territoire met en lumière un texte qui ne fait pas l'économie des apories de la société française contemporaine au risque d'en dérouter, mais aussi d'en séduire le lecteur ou une certaine critique en mal de réalisme. N'oublions pas qu'il incarne, de fait, un véritable phénomène médiatique et littéraire. D'où les réactions plutôt mitigées de la critique française et étrangère à l'égard de cet auteur et de son œuvre; une attitude qui, après le Goncourt, va plus loin que le simple bénéfice du doute et finit même par consacrer un écrivain et son style; un écrivain qui a définitivement su imposer sa carte et su marquer son territoire fictionnelle et stylistique.

Faut-il y voir le symptôme ou le reflet d'un malaise social profond, mais diffus, ou l'expression isolée d'un tempérament névrosé, enclin à la déprime et tenté par la contagion à la faveur du roman? "C'est vrai, je n'éprouve qu'un faible sentiment de solidarité à l'égard de l'espèce humaine..." (ib.,: 175), dit-il calmement en entretien à Jed Martin dans ce roman qui cherche à être plus que ça. On comprend mieux les réserves de Pierre Jourde, et de toute tendance de la critique envers ce "phénomène" extravagant et provocateur, ainsi que la réception très favorable dont bénéficiera le dernier roman houellebecquien une fois évacués le cynisme et la gratuité du scandale, comme si derrière la fiction et la caricature était également brossé un portrait somme toute fidèle, ou en tous cas crédible et sans fard, de nos sociétés et de leurs ambigüités: "Les romans de Michel Houellebecq dressent avec force le constat d'échec d'une civilisation, qui est peut-être aussi l'échec de l'humanité: la course au moi et à la différence est le moteur de l'apocalypse" (Jourde, 2002: 229). 


\section{Bibliographie}

ANDERSON, Perry (2005). La pensée tiède. Un regard critique sur la culture française. Paris: Seuil.

BADINTER, Elisabeth (1992). XY de l'identité masculine, Paris: Odile Jacob.

Blanckeman, Bruno (2000). Les récits indécidables: Jean Echenoz, Hervé Guibert, Pascal Quignard, Paris: Presses Universitaires du Septentrion.

ClEMENT, Murielle-Lucie (2003). Houellebecq, sperme et sang. Paris: L'Harmattan.

DEMONPION, Denis (2005). Houellebecq non autorisé. Enquête sur un phénomène. Paris: Maren Sell.

GANDILLOT, Thierry (2006). “Tournier: l'épris des mots” In: L'Express, 15 juin.

HouellebeCQ, Michel (1998). Les particules élémentaires, Paris: Flammarion (Coll. “J'ai lu”).

HouellebeCQ, Michel (2005). La possibilité d'une île. Paris: Fayard.

HouelleBECQ, Michel (2010). La carte et le territoire. Paris: Flammarion.

Huston, Nancy (2004). Professeurs de désespoir. Paris: Actes Sud.

JoURDE, Pierre (2002). La littérature sans estomac. Paris: L'esprit des péninsules.

JOURDE, Pierre (2005). Littérature \& authenticité. Le réel, le neutre, la fiction. Paris: L'Esprit des péninsules.

LINDENBERG, Daniel (2002). Rappel à l'ordre. Enquête sur les nouveaux réactionnaires.

Paris: Seuil / La République des idées.

LIPOVETSKY, Gilles (1983). L'ère du vide. Essai sur l'individualisme contemporain. Paris: Gallimard.

MORRISON, Donald (2008). Que reste-t-il de la culture française?. Paris: Denoël.

PATRICOLA, Jean-François (2005). Michel Houellebecq ou la provocation permanente. Paris: Ecriture.

PRIGENT, Christian (1991). Ceux qui merdRent. Paris: P.O.L.

VIART, Dominique / VERCIER, Bruno (2005). La littérature française au présent. Héritage, modernité, mutations, Paris: Bordas.

WEBER, Henri (2008). Faut-il liquider Mai 68?. Paris: Seuil. 